\title{
Iliac Crest Bone Grafting for the Management of Anterior Shoulder Instability in Patients with Glenoid Bone Loss: a Systematic Review of Contemporary Literature
}

Michael-Alexander Malahias ${ }^{1 *}$, Dimitrios Chytas², Vasileios Raoulis ${ }^{3}$, Efstathios Chronopoulos $^{2}$, Emmanouil Brilakis ${ }^{1}$ and Emmanouil Antonogiannakis ${ }^{1}$

\begin{abstract}
Background: A number of clinical trials have been published assessing the role of iliac crest bone grafting for the management of recurrent anterior instability with glenoid bone loss in contemporary practice. We therefore performed a systematic review of contemporary literature to examine the effect of iliac crest bone grafting on postoperative outcomes of these patients. Our hypothesis is that contemporary iliac crest bone block techniques are associated with low reoperation and complication rates combined with satisfactory functional results.

Methods: The US National Library of Medicine (PubMed/MEDLINE), the Cochrane Database of Systematic Reviews, and EMBASE were searched between January 2008 and December 2019 for relevant publications.

Results: Following the application of the inclusion-exclusion criteria, nine articles were found eligible for our analysis. In total, 261 patients (mean age range, 25.5-37.5 years; mean follow-up range, 20.6-42 months) were included in the studies of the current review. The mean modified Coleman score was 48.6 (range 37-65), indicating an overall low-to-moderate methodological quality. In the short term, the overall all-cause reoperation rate was $6.1 \%$, while the rate of recurrent instability was $4.8 \%$. The graft non-union rate was $2.2 \%$, while the rate of osteolysis, graft fracture, and infection was $0.4 \%, 0.9 \%$, and $1.7 \%$, respectively. Finally, hardware-related complications, such as screw breakage or symptomatic mechanical irritation around the screw insertion, occurred in $3.9 \%$ of the patients.

Conclusions: Iliac crest bone block techniques in contemporary practice are safe and effective in the short-term $(<$ 4 years) follow-up for the management of anterior shoulder instability with substantial glenoid bone deficiency. However, further studies of higher quality and longer follow-up are required to establish the therapeutic value of these techniques as well as to clarify whether there are differences in the outcomes of arthroscopic and open iliac crest bone block procedures.
\end{abstract}

Keywords: Glenoid bone block, Anterior shoulder instability, Anterior shoulder dislocation, Glenoid bone loss, lliac crest bone graft

\footnotetext{
*Correspondence: alexandermalahias@yahoo.gr

'3rd Orthopaedic Department, Hygeia Hospital, Erythrou Stavrou 4, Marousi,

15123 Athens, Greece

Full list of author information is available at the end of the article
} 


\section{Key Points}

- Iliac crest bone block techniques in contemporary practice might be safe and effective for the management of anterior shoulder instability with glenoid bone deficiency.

- Further studies of higher quality and longer followup are required to establish the therapeutic value of iliac crest bone block techniques in contemporary practice.

\section{Background}

Previous clinical and biomechanical studies have illustrated the importance of intact glenoid anatomy for shoulder stability $[1,2]$. In cases with substantial anterior-inferior glenoid osseous defects, isolated soft tissue repair techniques have been shown inadequate in restoring shoulder stability, since they have been related to high postoperative recurrence rates of up to $40 \%$ [3-7]. It is generally believed that cases with large glenoid bone defects would require a bone block augmentation technique to restore joint stability $[8,9]$. The Latarjet procedure with its various amendments has been the most commonly used bone block technique $[10,11]$. However, while the Latarjet procedure has proved to be reliable to manage recurrent anterior shoulder instability with large glenoid bone defects, there have been concerns of a high surgical complication rate associated with this procedure [12-14]. A large recent review reported an overall complication rate in the open Latarjet procedure of $15 \%$, with a $7 \%$ rate of unplanned reoperations [15].

An alternative option that has been used either for the revision of failed Latarjet procedures [16] or for the primary treatment of glenoid bone loss [10] is the EdenHybinnette technique. The initial procedure which has a 100-year history was based on the concepts of anatomic glenoid bony augmentation with a tibial autograft and capsulorrhaphy [17]. The traditional glenoid reconstruction has historically been associated with increased risk of postoperative degenerative changes [18].

Recently, several modifications in regard to the surgical approach, graft positioning and fixation, and the origin of the graft have been introduced [17]. In contrast to the modern techniques, in the original technique, Eden and Hybbinette placed the bone block inside the capsule at the anterior glenoid without any fixation and not flush with the glenoid, but as a mechanical dislocation barrier (not anatomically). Contemporary Eden-Hybinnette technique can be described by the use of an iliac crest bone block fixed with "low-profile" implants (buttons, sutures, J-shaped implant-free bone graft) through minimized open or arthroscopic procedures. A number of clinical trials have been published assessing the role of iliac crest bone grafting for the management of recurrent anterior instability with glenoid bone loss in contemporary practice. We therefore performed a systematic review of contemporary literature to examine the effect of iliac crest bone grafting technique on postoperative outcomes of these patients. Specifically, we aimed to answer the following questions: (1) what are the clinical and functional outcomes of the iliac crest bone block technique for the treatment of anterior shoulder instability with glenoid bone loss in contemporary practice? (2) Do contemporary iliac crest bone block techniques result in adequate bone graft healing, union, and osseous incorporation? (3) Do arthroscopic iliac crest bone block techniques result in similar clinical and radiographic outcomes compared to the respective open techniques? Our hypothesis is that contemporary iliac crest bone block techniques are associated with low reoperation and complication rates combined with satisfactory functional results.

\section{Methods \\ Search Criteria}

The US National Library of Medicine (PubMed/MEDLINE), Cochrane Database of Systematic Reviews, and EMBASE were searched (from December 2007 to December 2019) according to the Preferred Reporting Items for Systematic Reviews and Meta-Analyses (PRISMA) (Fig. 1) for publications utilizing keywords pertinent to anterior shoulder instability, glenoid bone loss, iliac crest bone graft, and clinical outcomes. Only abstracts that evaluated the utility of iliac crest bone grafting in anterior shoulder instability with glenoid bone loss were reviewed. The specific search terms are further shown in Table 1. To maximize the search, backward chaining of reference lists from retrieved papers was also undertaken. A preliminary assessment of only the titles and abstracts of the search results was initially performed. The second stage involved a careful review of the full-text publications.

\section{Inclusion and Exclusion Criteria}

The inclusion criteria were (1) studies describing human subjects of any age and sex, (2) studies that include a population of $>5$ patients who were originally treated with an iliac crest bone block technique for the reconstruction of glenoid bone insufficiency in patients with anterior shoulder instability, (3) studies that follow patients for a minimum of 18 months after surgery, and (4) studies that provide a clinical/functional and/or radiographic outcome measure (e.g., patient-reported outcome scores, postoperative complications, functional scores, range of motion, pain scale). The exclusion criteria were (1) review articles, (2) case studies with $\leq 5$ patients, (3) technical notes, (4) corrigenda, (5) editorial notes, (6) non-full-text articles, (7) studies without any clinical/functional or radiographic outcome, (8) studies in which patients were treated with coracoid transfer or 


\section{PRISMA 2009 Checklist}

\begin{tabular}{|c|c|c|c|}
\hline Section/topic & $\#$ & Checklist item & $\begin{array}{l}\text { Reported } \\
\text { on page \# }\end{array}$ \\
\hline \multicolumn{4}{|l|}{ TITLE } \\
\hline Title & 1 & Identify the report as a systematic review, meta-analysis, or both. & 1 \\
\hline \multicolumn{4}{|l|}{ ABSTRACT } \\
\hline Structured summary & 2 & $\begin{array}{l}\text { Provide a structured summary including, as applicable: background; objectives; data sources; study eligibility criteria, } \\
\text { participants, and interventions; study appraisal and synthesis methods; results; limitations; conclusions and } \\
\text { implications of key findings; systematic review registration number. }\end{array}$ & 2,3 \\
\hline \multicolumn{4}{|l|}{ INTRODUCTION } \\
\hline Rationale & 3 & Describe the rationale for the review in the context of what is already known. & 3,4 \\
\hline Objectives & 4 & $\begin{array}{l}\text { Provide an explicit statement of questions being addressed with reference to participants, interventions, comparisons, } \\
\text { outcomes, and study design (PICOS). }\end{array}$ & 4,5 \\
\hline \multicolumn{4}{|l|}{ METHODS } \\
\hline Protocol and registration & 5 & $\begin{array}{l}\text { Indicate if a review protocol exists, if and where it can be accessed (e.g., Web address), and, if available, provide } \\
\text { registration information including registration number. }\end{array}$ & 6 \\
\hline Eligibility criteria & 6 & $\begin{array}{l}\text { Specify study characteristics (e.g., PICOS, length of follow-up) and report characteristics (e.g., years considered, } \\
\text { language, publication status) used as criteria for eligibility, giving rationale. }\end{array}$ & 5 \\
\hline Information sources & 7 & $\begin{array}{l}\text { Describe all information sources (e.g., databases with dates of coverage, contact with study authors to identify } \\
\text { additional studies) in the search and date last searched. }\end{array}$ & 5 \\
\hline Search & 8 & $\begin{array}{l}\text { Present full electronic search strategy for at least one database, including any limits used, such that it could be } \\
\text { repeated. }\end{array}$ & 5,6 \\
\hline Study selection & 9 & $\begin{array}{l}\text { State the process for selecting studies (i.e., screening, eligibility, included in systematic review, and, if applicable, } \\
\text { included in the meta-analysis). }\end{array}$ & 6 \\
\hline Data collection process & 10 & $\begin{array}{l}\text { Describe method of data extraction from reports (e.g., piloted forms, independently, in duplicate) and any processes } \\
\text { for obtaining and confirming data from investigators. }\end{array}$ & 6 \\
\hline Data items & 11 & $\begin{array}{l}\text { List and define all variables for which data were sought (e.g., PICOS, funding sources) and any assumptions and } \\
\text { simplifications made. }\end{array}$ & 6 \\
\hline $\begin{array}{l}\text { Risk of bias in individual } \\
\text { studies }\end{array}$ & 12 & $\begin{array}{l}\text { Describe methods used for assessing risk of bias of individual studies (including specification of whether this was } \\
\text { done at the study or outcome level), and how this information is to be used in any data synthesis. }\end{array}$ & 6 \\
\hline Summary measures & 13 & State the principal summary measures (e.g., risk ratio, difference in means). & 6 \\
\hline Synthesis of results & 14 & $\begin{array}{l}\text { Describe the methods of handling data and combining results of studies, if done, including measures of consistency } \\
\left(\text { e.g., } I^{2} \text { ) for each meta-analysis. }\right.\end{array}$ & $\begin{array}{l}\text { N/A (no } \\
\text { meta- } \\
\text { analysis) }\end{array}$ \\
\hline Risk of bias across studies & 15 & $\begin{array}{l}\text { Specify any assessment of risk of bias that may affect the cumulative evidence (e.g., publication bias, selective } \\
\text { reporting within studies). }\end{array}$ & 6 \\
\hline Additional analyses & 16 & $\begin{array}{l}\text { Describe methods of additional analyses (e.g., sensitivity or subgroup analyses, meta-regression), if done, indicating } \\
\text { which were pre-specified. }\end{array}$ & $\begin{array}{l}\text { N/A (no } \\
\text { meta- } \\
\text { analysis) }\end{array}$ \\
\hline \multicolumn{4}{|l|}{ RESULTS } \\
\hline Study selection & 17 & $\begin{array}{l}\text { Give numbers of studies screened, assessed for eligibility, and included in the review, with reasons for exclusions at } \\
\text { each stage, ideally with a flow diagram. }\end{array}$ & 6 \\
\hline Study characteristics & 18 & $\begin{array}{l}\text { For each study, present characteristics for which data were extracted (e.g., study size, PICOS, follow-up period) and } \\
\text { provide the citations. }\end{array}$ & 7 \\
\hline Risk of bias within studies & 19 & Present data on risk of bias of each study and, if available, any outcome level assessment (see item 12). & 8,9 \\
\hline Results of individual studies & 20 & $\begin{array}{l}\text { For all outcomes considered (benefits or harms), present, for each study: (a) simple summary data for each } \\
\text { intervention group (b) effect estimates and confidence intervals, ideally with a forest plot. }\end{array}$ & $9-14$ \\
\hline Synthesis of results & 21 & Present results of each meta-analysis done, including confidence intervals and measures of consistency. & $\begin{array}{l}\text { N/A (no } \\
\text { meta- } \\
\text { analysis) }\end{array}$ \\
\hline Risk of bias across studies & 22 & Present results of any assessment of risk of bias across studies (see Item 15). & 8 \\
\hline Additional analysis & 23 & Give results of additional analyses, if done (e.g., sensitivity or subgroup analyses, meta-regression [see Item 16]). & $\begin{array}{l}\text { N/A (no } \\
\text { meta- } \\
\text { analysis) }\end{array}$ \\
\hline \multicolumn{4}{|l|}{ DISCUSSION } \\
\hline Summary of evidence & 24 & $\begin{array}{l}\text { Summarize the main findings including the strength of evidence for each main outcome; consider their relevance to } \\
\text { key groups (e.g., healthcare providers, users, and policy makers). }\end{array}$ & 15 \\
\hline Limitations & 25 & $\begin{array}{l}\text { Discuss limitations at study and outcome level (e.g., risk of bias), and at review-level (e.g., incomplete retrieval of } \\
\text { identified research, reporting bias). }\end{array}$ & 17 \\
\hline Conclusions & 26 & Provide a general interpretation of the results in the context of other evidence, and implications for future research. & 18 \\
\hline \multicolumn{4}{|l|}{ FUNDING } \\
\hline Funding & 27 & $\begin{array}{l}\text { Describe sources of funding for the systematic review and other support (e.g., supply of data); role of funders for the } \\
\text { systematic review. }\end{array}$ & 19 \\
\hline
\end{tabular}

Fig. 1 PRISMA 2009 checklist 
Table 1 Search criteria used

\begin{tabular}{lll}
\hline PubMed & Cochrane & EMBASE \\
\hline glenoid bone loss[tw] & $(($ "glenoid bone loss":ti,ab,kw OR & (("glenoid bone loss":ti,ab,de,tn,kw OR \\
OR glenoid defects[tw] & "glenoid defects":ti,ab,kw OR & "glenoid defects":ti,ab,de,tn,kw OR \\
OR glenoid defect[tw] & "glenoid defect":ti,ab,kw)) & "glenoid defect":ti,ab,de,tn,kw)) \\
AND & AND & AND \\
(Anterior[tw] AND "Shoulder dislocation"[Mesh]) & ((Anterior:ti,ab,kw AND [mh & ((Anterior:ti,ab,de,tn,kw AND 'Shoulder \\
OR Anterior shoulder instability[tw] & "Shoulder dislocation"]) OR "Anterior & dislocation'/exp) OR "Anterior shoulder \\
OR Anterior shoulder dislocation[tw] & shoulder instability":ti,ab,kw OR & instability":ti,ab,de,tn,kw OR "Anterior \\
OR Anteroinferior Shoulder Instability[tw] & "Anterior shoulder dislocation":ti,ab, & shoulder dislocation":ti,ab,de,tn,kw OR \\
OR shoulder instability[tw] & kw OR "Anteroinferior Shoulder & "Anteroinferior Shoulder Instability":ti,ab, \\
AND & Instability":ti,ab,kw OR "shoulder & de,tn,kw OR "shoulder instability":ti,ab, \\
iliac crest bone grafting[tw] & instability":ti,ab,kw)) & de,tn,kw)) \\
OR iliac crest bone graft[tw] & AND & AND \\
OR iliac crest bone grafts[tw] & ("iliac crest bone grafting":ti,ab,kw & ("iliac crest bone grafting":ti,ab,de,tn,kw \\
OR iliac graft[tw] & OR "iliac crest bone graft":ti,ab,kw & OR "iliac crest bone graft":ti,ab,de,tn,kw \\
OR iliac grafts[tw] & OR "iliac crest bone grafts":ti,ab,kw & OR "iliac crest bone grafts":ti,ab,de,tn,kw \\
OR glenoid bone block[tw] & OR "iliac graft":ti,ab,kw OR "iliac & OR "iliac graft":ti,ab,de,tn,kw OR "iliac \\
OR Arthroscopic bone block grafting[tw] & grafts":ti,ab,kw OR "glenoid bone & grafts":ti,ab,,de,tn,kw OR "glenoid bone \\
OR J-bone graft[tw] & block":ti,ab,kw OR "Arthroscopic & block":ti,ab,de,tn,kw OR "Arthroscopic \\
& bone block grafting":ti,ab,kw & bone block grafting":ti,ab,de,tn,kw OR \\
& OR "J-bone graft":ti,ab,kw) & "J-bone graft":ti,ab,de,tn,kw) \\
\hline
\end{tabular}

other than iliac crest types of bone graft or soft-tissue surgery, (9) studies in which no subjects underwent iliac crest bone block technique, (10) non-English language publications, (11) studies published before December 2, 2007, or after December 2, 2019, (12) studies including patients who were operated on before 2000, and (13) studies with follow-up $<18$ months.

\section{Data Collection}

Two authors independently conducted the search [MM, DC]. To maximize the search, backward chaining of reference lists from retrieved papers was also undertaken. A preliminary assessment of only the titles and abstracts of the search results was initially performed. The second stage involved a careful review of the full-text publications. All authors compiled a list of articles not excluded after application of the inclusion and exclusion criteria. Discrepancies between the authors were resolved by discussion. In cases of disagreement, the senior author (EA) had the final decision.

During initial review of the data, the following information was collected for each study: title, author, year published, study design, number of patients, number of operated joints, sex, type of interventional treatment performed, type of graft, type of implant used, success percentage of treatment (free from recurrence of instability), reoperation rate, complication rate, return to sports, range of motion, clinical/ functional subjective scores, and progression of glenohumeral osteoarthritis.

The methodological quality of each study and the different types of detected bias were assessed independently by each reviewer [MM, DC] with the use of modified Coleman methodology score $[19,20]$. Selective reporting bias like publication bias was not included in the assessment. The primary outcome measure was the free-from- recurrence-of-instability rate, and the clinical, functional, and radiographic outcomes. Secondary outcome was the quality assessment of the studies with the use of the modified Coleman methodology score.

\section{Results}

The literature search identified 76 abstracts that were examined to determine the efficacy of the iliac crest bone block technique for the management of glenoid bone loss in patients with anterior shoulder instability (Fig. 1). Following the application of the inclusionexclusion criteria, nine articles were found eligible for our systematic analysis [21-28]. A summary flowchart of our literature search according to PRISMA guidelines can be found in Fig. 2.

\section{Demographics}

Totally, 261 patients (217 males, 44 females) were included in the studies of the current review. From them, 231 patients underwent placement of iliac crest graft (40 with an open procedure, 191 with the arthroscopic procedure) and 30 patients underwent the Latarjet procedure. Their mean age ranged from 25.5 [21] to 37.5 years old [22]. The mean follow-up ranged from 20.6 months [23] to 42 months [25] (Table 2).

\section{Level of evidence and quality of the studies}

One out of nine studies of this review (11.1\%) had a level of evidence III [26], one (11.1\%) had a level of evidence I [24], while all other studies (77.8\%) had a level of evidence IV (Table 2).

Apart from the study by Moroder et al. [24], all the other papers were characterized by selection, detection, and performance bias, while all apart from three studies $[24,27,29](33.3 \%)$ declared that some of their authors 


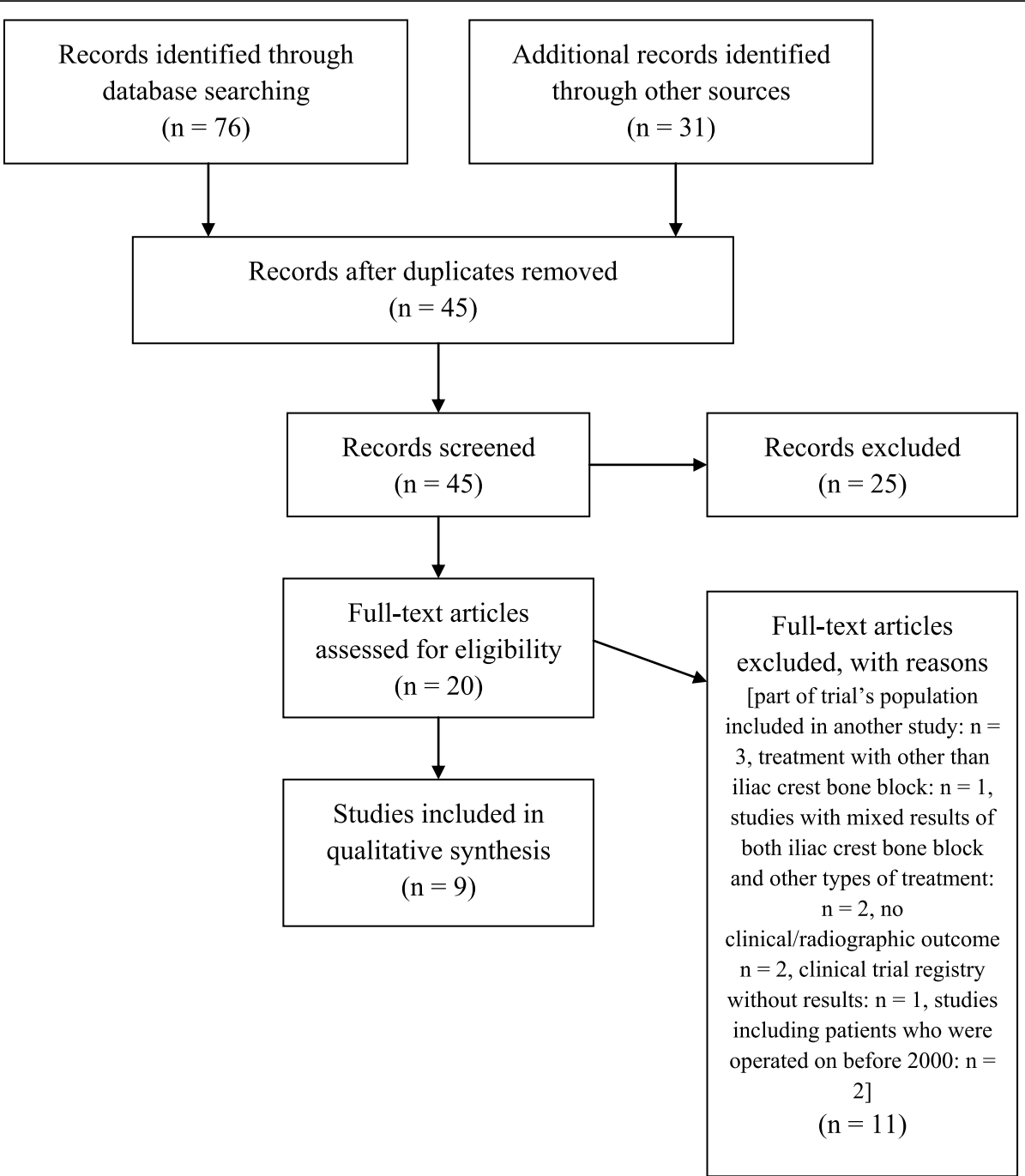

Fig. 2 Flow chart of study selection according to PRISMA guidelines for reporting systematic reviews and meta-analyses

had a relevant conflict of interests. The mean modified Coleman methodology score was 48.6, while it ranged from 37 [26] to 65 [27] indicating an overall low-tomoderate methodological quality (Table 2).

\section{Type of Graft and Fixation}

Seven studies (77.8\%) described the use of autologous iliac crest grafts, whereas the remaining two studies (22.2\%) made use of iliac crest allografts [21, 27]. Four studies $(44.4 \%)$ used screws for the bone block fixation $[22,23,25,29]$, while one study $(11.1 \%)$ used endobuttons [21] and the other one (11.1\%) sutures [27]. Finally, there were two studies $(22.2 \%)$ that examined the use of a J-shaped formed iliac crest bone block that fixed without any implants (implant-free technique) $[26,28]$ and one study [24] (11.1\%) that comprised two groups: in the first one, the coracoid graft was fixed with screws, while in the second one, a J-shaped formed iliac crest bone block was fixed without any implants (Table 2).

\section{Clinical/functional subjective scores}

Eight out of the nine studies $(88.9 \%)$ reported clinical or functional subjective outcome variables [21-25, 27-29]. All mean postoperative clinical/functional subjective scores which were reported in the studies of this review were significantly improved compared to the respective mean preoperative values. The Rowe score was the most commonly used, since it was documented in seven studies (77.8 \% of all studies) [21-24, 27-29]. The Western Ontario Shoulder Instability Index (WOSI) was used in five studies (55.6\%) [22-25, 29]. The Constant score was utilized in four studies (44.4\% of all studies) [23, 25, 28, 29] as well as the subjective shoulder value (SSV) [21, 23, 24, 28]. The Walch-Duplay score was measured in three studies $(33.3 \%)[21,22,29]$. The visual analog scale 


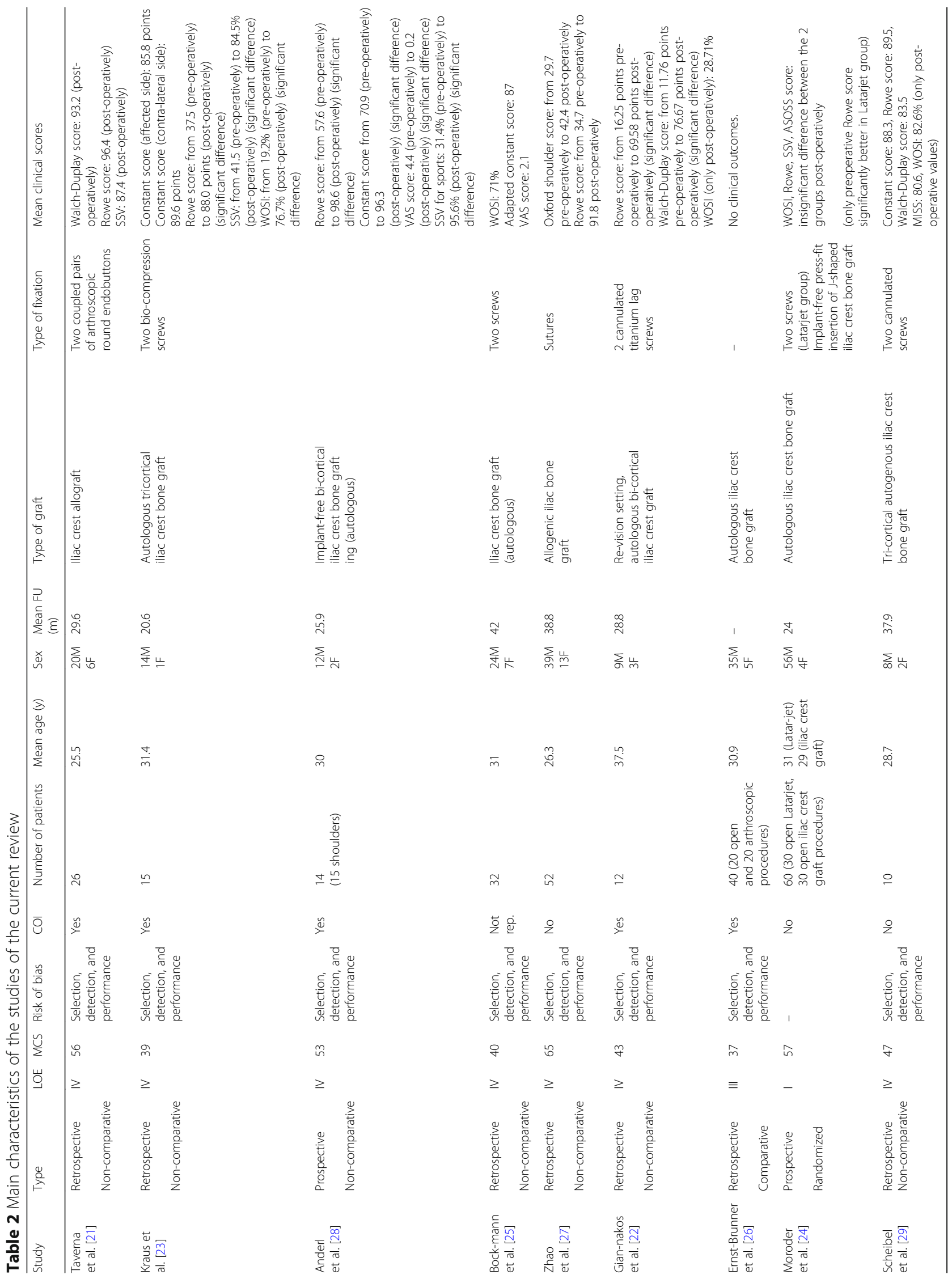


(VAS) score was measured in two studies $(22.2 \%$ of all studies) $[25,28]$. Finally, the Oxford Shoulder Score was examined in one study (11.1\% of all) [27], the Athletic Shoulder Outcome Scoring System (ASOSS) in one study (11.1\%) [24], and the Melbourne Instability Shoulder Score (MISS) in one study (11.1\%) [29] (Table 2).

\section{Range of Motion}

Seven out of the nine studies (77.8\%) dealt with the range of motion (ROM) of their patients' operated shoulder [22-25, 27-29]. Kraus et al. [23] did not find significant differences in any of the different elements of postoperative ROM compared to the healthy contralateral side. Scheibel et al. [29] found that the mean hand-to-back distance of the affected side (achieved actively during the liftoff test) was significantly inferior to that of the healthy side. In addition, Anderl et al. noted that all elements of ROM were significantly improved compared to the preoperative respective values [28]. Bockmann et al. reported that their patients achieved full ROM in the abduction and external rotation post-surgery [25], while Zhao et al. noted that almost all patients of their cohort had normal postoperative ROM [27].

\section{Clinical/Functional Subjective Scores}

Eight out of the nine studies (88.9\%) reported clinical or functional subjective outcome variables [21-25, 27-29]. All mean postoperative clinical/functional subjective scores which were reported in the studies of this review were significantly improved compared to the respective mean preoperative values. The Rowe score was the most commonly used, since it was documented in seven studies (77.8\% of all studies) [21-24, 27-29]. The Western Ontario Shoulder Instability Index (WOSI) was used in five studies $(55.6 \%)$ [22-25, 29]. The constant score was utilized in four studies (44.4\% of all studies) [23, 25, 28, 29] as well as the SSV [21, 23, 24, 28]. The WalchDuplay score was measured in three studies (33.3\%) [21, $22,29]$. The VAS score was measured in two studies (22.2\% of all studies) [25, 28]. Finally, the Oxford Shoulder Score was examined in one study (11.1\% of all) [27], the Athletic Shoulder Outcome Scoring System (ASOSS) in one study (11.1\%) [24], and the MISS in one study (11.1\%) [29] (Table 2).

\section{Return to Athletic Activities}

Three out of the nine studies (33.3\%) documented return to sports after surgery as one of the success rate criteria $[21,22,28]$. Both Taverna et al. [21] and Giannakos et al. [22] found that two thirds of their patients who played sports before injury (66.7\%) returned to their pre-injury level of athletic activity. In addition, Anderl et al. noted that all patients returned to their pre-injury level of athletic activity within 6 months after surgery [28].

\section{Complications}

The overall all-cause reoperation rate was $6.1 \%$ (14 out of 231 patients), while the overall complication rate was $19.9 \%$ (46 out of 231 patients).

\section{Recurrent Instability (Dislocation, Subluxation, Positive Apprehension Test)}

There were 11 cases of recurrent dislocation or subluxation (out of 231 patients; 4.8\%). Three of these 11 cases were re-operated, whereas the other eight cases were treated conservatively. In addition, there were 11 cases of persistent positive apprehension test (4.8\% of all patients), one of which underwent plication of the capsule [23].

\section{Osteolysis and Non-union of the Graft}

In total, two out of the nine studies of the current review $(22.2 \%)$ reported cases of non-union or osteolysis of the bone graft [21, 22]. Overall, there were five cases (out of 231 patients; $2.2 \%$ ) of non-union and one case of osteolysis (0.4\%).

\section{Infection Rate}

Two out of the nine studies (22.2\%) reported in total four postoperative cases of infection [24, 25] (four patients out of 231 , rate $1.7 \%$ ).

\section{Hardware-Related Complications}

The rate of hardware-related complications was 3.9\% (9 out of 231 cases). Bockmann et al. reported that two out of the 32 patients $(6.3 \%$ of their patient cohort) experienced mechanical irritation around the screw insertion sites generating persistent pain [25]. These patients were successfully treated with arthroscopic removal of the screw [25]. Zhao et al. found two out of the 52 cases (3.8\% of their patient cohort) with posterior-inferior penetration of the glenoid by the tip of the anchors [27]. In addition, Giannakos et al. noted that four out of the 12 patients (33.3\% of their patient cohort) required hardware removal due to possible contact between humeral head and screws [22]. Moreover, one patient was radiographically diagnosed with screw breakage which did not require revision surgery [22].

\section{Other Complications}

Other complications, which were not previously described, were diagnosed in 16 patients (6.9\% of all patients). Neurological hypoesthesia at the donor site was observed in 10 patients (out of 128 with iliac crest harvesting, 7.8\%) and graft fracture in two cases (out of 231, 0.9\%). Finally, one study reported postoperative hematoma in two out of the 26 patients $(7.7 \%)$ which resolved spontaneously. 


\section{Postoperative Progression of Osteoarthritis}

Six out of the nine studies of the present review $(66.7 \%)$ assessed the presence of glenohumeral osteoarthritis [21-23, 27-29]. In total, nine cases (out of 231, 3.9\%) of the progression of glenohumeral osteoarthritis were noted.

\section{Arthroscopic Versus Open Iliac Crest Bone Block Technique}

One out of the nine studies (11.1\%) compared the radiographic outcome of arthroscopic versus open glenoid reconstruction with iliac crest bone block graft [26]. The covered defect size was significantly different amongst groups (95\% in the arthroscopic group, $98 \%$ in the open group) [26]. The arthroscopic group showed a significantly steeper mean impaction angle $\left(34.8^{\circ}\right)$ and significantly increased mean medial offset $(6.6 \mathrm{~mm})$ compared to the open group (mean impaction angle of $26.9^{\circ}$, mean medial offset $5.4 \mathrm{~mm}$ ) [26]. Finally, the mean difference in the mediolateral step formation amongst groups was not significant $(2.9 \mathrm{~mm}$ in the arthroscopic group and $3.2 \mathrm{~mm}$ in the open group) [26].

lliac Crest Bone Block Versus Coracoid Transfer Technique One out of the nine studies of the current review (11.1\%) compared the outcomes of iliac crest bone graft (open) technique and open coracoid transfer technique (Latarjet) for the treatment of anterior shoulder instability with the glenoid bone loss [24]. Moroder et al. [24] did not find any significant differences in the failure rates of the Latarjet group and the iliac crest graft group. The two procedures did not differ significantly in WOSI, Rowe score, SSV, or ASOSS score at any follow-up time point, while internal rotation was significantly higher in the iliac crest graft group compared to the Latarjet group. Furthermore, there were not any significant differences between the two groups in postoperative pain, satisfaction, strength, abduction, and external rotation at the final follow-up. Finally, the defect area was significantly lower in the iliac crest graft group at final followup.

\section{Iliac Crest Bone Autograft Versus lliac Crest Bone Allograft in Glenoid Reconstruction}

No study was found to compare the outcomes of iliac crest bone autograft versus iliac crest bone allograft in the treatment of anterior shoulder instability with glenoid bone loss.

\section{Discussion}

A trend exists toward increased utilization of bone-block stabilization for the treatment of shoulder instability among recently trained orthopedic surgeons [30]. With the better understanding of the role of "engaging" Hill-
Sachs lesions in glenohumeral biomechanics and the specific indications for bone-block glenoid reconstruction, in combination with the use of meticulous preoperative planning, advanced imaging (3D reconstruction) of the glenohumeral bone defects, minimally invasive surgical techniques, sophisticated implants, and individualized evidence-based rehabilitation protocols, glenoid bone block augmentation surgery has rapidly evolved over the last few years [30]. Although a number of studies have been recently published in the literature, none of the previous reviews examined in a systematic manner the outcomes of contemporary-only iliac crest bone block techniques $[17,31,32]$. To address this point, we conducted a systematic review of contemporary literature including publications from the last 12 years.

The most important finding of this analysis was that, regardless of the fixation method, iliac crest bone block grafting was a satisfactory treatment for cases with recurrent anterior instability and substantial glenoid bone loss, since it resulted in low all-cause reoperation (6.1\%) rate. The rates of recurrent instability (4.8\%) and positive anterior apprehension test $(4.8 \%)$ were also very low in the short term. Furthermore, regardless of the graft type (bicortical or tricortical autograft, J-shaped autograft, allograft), non-union (2.2\%), osteolysis of the graft $(0.4 \%)$, graft fracture $(0.9 \%)$, or infection $(1.7 \%)$ were very rarely noticed. Finally, hardware-related complications, such as screw breakage or symptomatic mechanical irritation around the screw insertion, were not common (3.9\%). Based on these findings, we suggest that the contemporary use of iliac crest bone grafting is safe and effective in the short term for the management of anterior shoulder instability with substantial glenoid bone deficiency.

Whereas historical goals centered on the stable reduction and prevention of recurrent dislocation, current standards of success are predicated on the restoration of motion and strength and the return to functional activities, including competitive athletics [33]. In our analysis, the use of iliac crest bone graft resulted in significantly improved functional scores after surgery. In addition, postoperative ROM was significantly improved with none to minimal rotational loss. Finally, it was shown that the majority of patients who were treated with iliac crest bone grafting returned to their pre-injury level of athletic activities [21, 22, 28].

Recurrent glenohumeral instability represents a treatment challenge for orthopedic surgeons as it not only has the potential to result in a subsequent surgery, therapy, and missed activity time, but also has been associated in the past with long-term degenerative joint changes [34]. Although none of the studies included in our analysis showed any progression of osteoarthritis after short- to mid-term follow-up, we did not find any study to examine this variable in the long term. Based 
on this finding, it could be supported that contemporary iliac crest bone block techniques are not associated with short-term degenerative joint changes, but further studies are required to assess its long-term effect.

Iliac crest bone blocks that have been used for glenoid reconstruction were either autografts or allografts. Although the iliac crest is a convenient source of customizable autologous bone grafts, it has been associated with a substantial risk of chronic degenerative changes in the glenohumeral joint as well as immediate and, sometimes, persistent pain at the donor site [35, 36]. Allogeneic osteochondral iliac crest grafts were introduced to minimize the risk of arthropathy and donor site morbidity [18]. Although concerns have been raised regarding potential early resorption and inadequate osseointegration of the graft, an allograft-focused review showed that allograft reconstruction for glenoid bone loss provided excellent clinical outcomes, low rates of recurrent instability, and high osseous incorporation rates with no evidence of graft resorption [37].

In our analysis, both iliac crest autograft and allograft resulted in excellent survival rates and high osseous incorporation, although there was no study to directly compare them. Neither allograft nor autograft resorption occurred in the patient cohorts of our review. Moreover, no cases of osteoarthritic progression were found either with the use of autograft or allograft. Problems related to the donor site of the autograft, such as hypoesthesia, wound-related complications, or persistent pain, were very rare. Taking into consideration these findings, we feel that further research of higher quality should be done to lead to definite conclusions regarding the use of iliac crest allografts for cases requiring glenoid reconstruction, when iliac crest autografts of good quality are available.

There was only one study [26] to compare arthroscopic versus open iliac crest bone grafting procedures reporting that the open group was associated with increased coverage of the glenoid defect $(95 \%$ in the arthroscopic group vs. $98 \%$ in the open group), steeper mean impaction angle, and increased mean medial offset compared to the arthroscopic group. However, the clinical relevance of these radiographic findings was unclear, since the clinical outcomes of both techniques were excellent.

Furthermore, there was only one study to compare open iliac crest bone grafting and Latarjet technique at 2 years follow-up [24]. In this bicentric prospective randomized study of 60 patients with anterior shoulder instability and glenoid bone loss, Moroder et al. [24] found no significant differences in failure rate, ROM, functional scores, satisfaction, and strength between the two procedures. However, further studies are required to confirm that the outcome of iliac crest bone grafting does not significantly differ from the glenoid transfer.
This review was not without limitations. Most of the studies included in this analysis were single series of patients without any control group. All apart from one study [24] were of low level of evidence (either III or IV) with selection, detection, and performance bias that might have affected the validity of the outcomes reported. In addition, all except for three studies [24, 27, 29] (33.3\%) declared that some of their authors had relevant conflict of interests. The "quality assessment" of the studies for methodological deficiencies, as a common alternative to "risk of bias," was examined by the modified Coleman methodology score [20], and it was to be found low to moderate. The study design, including the type of graft, type of fixation, follow-up, and type of surgery (open or arthroscopic), was relatively heterogeneous. In addition, there was a complete lack of mid- or long-term results. However, all studies examined a specific surgical technique (iliac crest bone block), which has gained increasing attention among physicians in recent years (almost all publications were from 2014 to 2018). In addition, the results of all studies were towards the same direction, since they all depicted that iliac crest bone block resulted in satisfactory clinical outcomes with low failure rates.

\section{Conclusions}

Iliac crest bone block techniques in contemporary practice are safe and effective in the short-term $(<4$ years $)$ followup for the management of anterior shoulder instability with substantial glenoid bone deficiency. However, further studies of higher quality and longer follow-up are required to establish the therapeutic value of these techniques as well as to clarify whether there are differences in the outcomes of arthroscopic and open iliac crest bone block procedures.

\section{Abbreviations}

ASOSS: Athletic Shoulder Outcome Scoring System; COI: Conflict of interest: F: Females; FU: Follow-up; LOE: Level of evidence; M: Males; m: Months; MCS: Modified Coleman Score; MISS: Melbourne Instability Shoulder Score; Not rep.: Not reported; PRISMA: Preferred Reporting Items for Systematic reviews and Meta-analyses; ROM: Range of motion; SSV: Subjective shoulder value; TW: Text word; VAS: Visual analog scale; WOSI: Western Ontario Shoulder Instability Index; y: Years

\section{Acknowledgements}

None declared

\section{Authors' Contributions}

MAM conducted the research protocol, assessed the data of the review independently from other reviewers, and wrote the manuscript as the main author. DC gathered the data and assessed them independently from other reviewers. VR wrote a part of the manuscript. EC critically revised and edited the manuscript. EB assisted in the critical revision of the manuscript. EA supervised as senior author of the whole project, revised critically, and edited the manuscript. All authors read and approved the final version of the manuscript.

Funding

No sources of funding were used to assist in the preparation of this review. 


\section{Availability of Data and Materials}

Data sharing not applicable to this article as no datasets were generated or analyzed during the current study.

\section{Ethics Approval and Consent to Participate}

Not applicable (review article without involving animals, human participants, human data, or human tissue).

\section{Consent for Publication}

Not applicable.

\section{Competing Interests}

The authors, Michael-Alexander Malahias, Dimitrios Chytas, Vasileios Raoulis, Efstathios Chronopoulos, Emmanouil Brilakis, and Emmanouil

Antonogiannakis, declare that they have no competing interests relevant to the content of this review.

\section{Author details}

'3rd Orthopaedic Department, Hygeia Hospital, Erythrou Stavrou 4, Marousi, 15123 Athens, Greece. ${ }^{2}$ 2nd Orthopaedic Department, School of Medicine, National \& Kapodistrian University of Athens, Agias Olgas 3, Nea Ionia, 14233 Athens, Greece. ${ }^{3}$ Department of Orthopaedic Surgery, Faculty of Medicine, School of Health Sciences, University of Thessaly, Larissa, Greece.

\section{Received: 26 July 2019 Accepted: 27 January 2020}

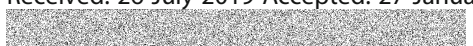

\section{References}

1. Burkhart SS, De Beer JF. Traumatic glenohumeral bone defects and their relationship to failure of arthroscopic Bankart repairs: significance of the inverted-pear glenoid and the humeral engaging Hill-Sachs lesion. Arthroscopy. 2000;16(7):677-94.

2. Itoi $E$, Lee $S B$, Berglund $L J$, Berge $L L$, An KN. The effect of a glenoid defect on anteroinferior stability of the shoulder after Bankart repair: a cadaveric study. J Bone Joint Surg Am. 2000;82(1):35-46.

3. Castagna A, Markopoulos N, Conti M, Rose GD, Papadakou E, Garofalo R. Arthroscopic Bankart suture-anchor repair: radiological and clinical outcome at minimum 10 years of follow-up. Am J Sports Med. 2010;38(10):2012-6.

4. Sugaya H, Moriishi J, Kanisawa I, Tsuchiya A. Arthroscopic osseous Bankart repair for chronic recurrent traumatic anterior glenohumeral instability. J Bone Joint Surg Am. 2005;87(8):1752-60.

5. Elmlund A, Kartus C, Sernert N, Hultenheim I, Ejerhed L. A long-term clinical follow-up study after arthroscopic intra-articular Bankart repair using absorbable tacks. Knee Surg Sports Traumatol Arthrosc. 2008;16(7):707-12.

6. Tauber M, Resch H, Forstner R, Raffl M, Schauer J. Reasons for failure after surgical repair of anterior shoulder instability. J Shoulder Elb Surg. 2004; 13(3):279-85.

7. Taverna E, Garavaglia G, Ufenast H, D'Ambrosi R. Arthroscopic treatment of glenoid bone loss. Knee Surg Sports Traumatol Arthrosc. 2016;24(2):546-56. https://doi.org/10.1007/s00167-015-3893-0

8. Longo UG, Loppini M, Rizzello G, Ciuffreda M, Maffulli N, Denaro V. Latarjet, Bristow, and Eden-Hybinette procedures for anterior shoulder dislocation: systematic review and quantitative synthesis of the literature. Arthroscopy. 2014;30(9):1184-211. https://doi.org/10.1016/..arthro.2014.04.005.

9. Boileau P, Mercier N, Old J. Arthroscopic Bankart-Bristow-Latarjet (2B3) Procedure: how to do it and tricks to make it easier and safe. Orthop Clin North Am. 2010;41(3):381-92. https://doi.org/10.1016/j.ocl.2010.03.005.

10. Hurley ET, Jamal MS, Ali ZS, Montgomery C, Pauzenberger L, Mullett $H$. Long-term outcomes of the Latarjet procedure for anterior shoulder instability: a systematic review of studies at 10-year follow-up. J Shoulder Elb Surg. 2019;28(2):e33-9. https://doi.org/10.1016/j.jse.2018.08.028.

11. Malahias MA, Fandridis E, Chytas D, Chronopulos E, Brilakis E, Antonogiannakis E. Arthroscopic versus open Latarjet: a step-by-step comprehensive and systematic review. Eur J Orthop Surg Traumatol. 2019, 29(5):957-66. https://doi.org/10.1007/s00590-019-02398-3.

12. Bedeir YH, Schumaier AP, Grawe BM. The failed Latarjet procedure: evaluation, treatment, and outcomes. JBJS Rev. 2018;6(10):e10. https://doi. org/10.2106/JBJS.RWW.18.00002

13. Frank RM, Gregory B, O'Brien M, Bernardoni E, Verma NN, Cole BJ, Nicholson GP, Romeo AA. Ninety-day complications following the Latarjet procedure. $J$ Shoulder Elb Surg. 2019;28(1):88-94. https://doi.org/10.1016/j.jse.2018.06.022.
14. Ranalletta M, Tanoira I, Bertona A, Maignon G, Bongiovanni S, Rossi LA. Autologous tricortical iliac bone graft for failed Latarjet procedures. Arthrosc Tech. 2019;8(3):e283-9. https://doi.org/10.1016/j.eats.2018.11.002.

15. Domos P, Lunini E, Walch G. Contraindications and complications of the Latarjet procedure. Should Elb. 2018;10(1):15-24.

16. Tytherleigh-Strong GM, Morrissey DI. Failed Latarjet procedure treated with a revision bone block stabilization using a suture-button fixation. J Shoulder Elb Surg. 2017;26(4):e102-7. https://doi.org/10.1016/j.jse.2017.01.004.

17. Villatte G, Spurr S, Broden C, Martins A, Emery R, Reilly P. The EdenHybbinette procedure is one hundred years old! A historical view of the concept and its evolutions. Int Orthop. 2018;42(10):2491-5. https://doi.org/ 10.1007/s00264-018-3970-3.

18. Willemot $L B$, Elhassan $B T$, Verborgt $O$. Bony reconstruction of the anterior glenoid rim. J Am Acad Orthop Surg. 2018;26(10):e207-18. https://doi.org/ 10.5435/JAAOS-D-16-00649.

19. Viswanathan M, Ansari MT, Berkman ND, et al. Assessing the risk of bias of individual studies in systematic reviews of health care interventions. Methods Guide for Effectiveness and Comparative Effectiveness Reviews [Internet]. Rockville (MD): Agency for Healthcare Research and Quality (US); 2008-.2012 Mar 8.

20. Sambandam SN, Gul A, Priyanka P. Analysis of methodological deficiencies of studies reporting surgical outcome following cemented total-joint arthroplasty of trapezio-metacarpal joint of the thumb. Int Orthop. 2007;31(5):639-45.

21. Taverna E, Garavaglia G, Perfetti C, Ufenast H, Sconfienza LM, Guarrella V. An arthroscopic bone block procedure is effective in restoring stability, allowing return to sports in cases of glenohumeral instability with glenoid bone deficiency. Knee Surg Sports Traumatol Arthrosc. 2018. https://doi.org/ 10.1007/s00167-018-4921-7.

22. Giannakos A, Vezeridis PS, Schwartz DG, Jany R, Lafosse L. All-arthroscopic revision Eden-Hybinette procedure for failed instability surgery: technique and preliminary results. Arthroscopy. 2017;33(1):39-48. https://doi.org/10. 1016/j.arthro.2016.05.021.

23. Kraus N, Amphansap T, Gerhardt C, Scheibel M. Arthroscopic anatomic glenoid reconstruction using an autologous iliac crest bone grafting technique. J Shoulder Elb Surg. 2014;23(11):1700-8. https://doi.org/10.1016/j. jse.2014.03.004

24. Moroder P, Schulz E, Wierer G, Auffarth A, Habermeyer P, Resch H, Tauber M. Neer Award 2019: Latarjet procedure vs. iliac crest bone graft transfer for treatment of anterior shoulder instability with glenoid bone loss: a prospective randomized trial. J Shoulder Elb Surg. 2019;28(7):1298-307. https://doi.org/10.1016/j.jse.2019.03.035.

25. Bockmann B, Venjakob AJ, Reichwein F, Hagenacker M, Nebelung W. Midterm clinical results of an arthroscopic glenoid rim reconstruction technique for recurrent anterior shoulder instability. Arch Orthop Trauma Surg. 2018; 138(11):1557-62. https://doi.org/10.1007/s00402-018-2964-3.

26. Ernstbrunner L, Plachel F, Heuberer P, et al. Arthroscopic versus open iliac crest bone grafting in recurrent anterior shoulder instability with glenoid bone loss: a computed tomography-based quantitative assessment. Arthroscopy. 2018;34(2):352-9. https://doi.org/10.1016/j.arthro.2017.07.034.

27. Zhao J, Huangfu X, Yang X, Xie G, Xu C. Arthroscopic glenoid bone grafting with nonrigid fixation for anterior shoulder instability: 52 patients with 2- to 5-year follow-up. Am J Sports Med. 2014;42(4):831-9. https://doi.org/10. 1177/0363546513519227.

28. Anderl W, Pauzenberger L, Laky B, Kriegleder B, Heuberer PR. Arthroscopic implant-free bone grafting for shoulder instability with glenoid bone loss: clinical and radiological outcome at a minimum 2-year follow-up. Am J Sports Med. 2016;44(5):1137-45. https://doi.org/10.1177/0363546515625283.

29. Scheibel M, Nikulka C, Dick A, Schroeder RJ, Gerber Popp A, Haas NP. Autogenous bone grafting for chronic anteroinferior glenoid defects via a complete subscapularis tenotomy approach. Arch Orthop Trauma Surg. 2008;128(11):1317-25. https://doi.org/10.1007/s00402-007-0560-z.

30. Degen RM, Camp CL, Werner BC, Dines DM, Dines JS. Trends in bone-block augmentation among recently trained orthopaedic surgeons treating anterior shoulder instability. J Bone Joint Surg Am. 2016;98(13):e56. https:// doi.org/10.2106/JBJS.15.01478.

31. Nzeako O, Bakti N, Bawale R, Singh B. Bone block procedures for glenohumeral joint instability. J Clin Orthop Trauma. 2019;10(2):231-5. https://doi.org/10.1016/j.jcot.2018.10.002.

32. Ramhamadany E, Modi CS. Current concepts in the management of recurrent anterior gleno-humeral joint instability with bone loss. World Orthop. 2016;7(6):343-54. https://doi.org/10.5312/wjo.v7.66.343. 
33. Chen AL, Hunt SA, Hawkins RJ, Zuckerman JD. Management of bone loss associated with recurrent anterior glenohumeral instability. Am J Sports Med. 2005;33(6):912-25.

34. Donohue MA, Mauntel TC, Dickens JF. Recurrent shoulder instability after primary Bankart repair. Sports Med Arthrosc Rev. 2017;25(3):123-30. https:// doi.org/10.1097/JSA.0000000000000159.

35. Moroder P, Blocher M, Auffarth A, et al. Clinical and computed tomographyradiologic outcome after bony glenoid augmentation in recurrent anterior shoulder instability without significant glenoid bone loss. J Shoulder Elb Surg. 2014;23(3):420-426.44.

36. Myeroff C, Archdeacon M. Autogenous bone graft: donor sites and techniques. J Bone Joint Surg Am. 2011;93(23):2227-36.

37. Sayegh ET, Mascarenhas R, Chalmers PN, Cole BJ, Verma NN, Romeo AA. Allograft reconstruction for glenoid bone loss in glenohumeral instability: a systematic review. Arthroscopy. 2014;30(12):1642-9. https://doi.org/10.1016/j. arthro.2014.05.007.

\section{Publisher's Note}

Springer Nature remains neutral with regard to jurisdictional claims in published maps and institutional affiliations.

\section{Submit your manuscript to a SpringerOpen ${ }^{\circ}$ journal and benefit from:}

- Convenient online submission

- Rigorous peer review

- Open access: articles freely available online

- High visibility within the field

Retaining the copyright to your article

Submit your next manuscript at $\boldsymbol{\wedge}$ springeropen.com 